\title{
EPIMORPHISMS AND MONOMORPHISMS IN HOMOTOPY
}

\author{
JERZY DYDAK
}

(Communicated by James E. West)

ABSTRACT. The main result of this note is the following:

Theorem A. If $f: X \rightarrow Y$ is an epimorphism of $\mathscr{H} \mathscr{C} \mathscr{W}^{*}$, the homotopy category of pointed path-connected CW-spaces, and $\pi_{1}(f): \pi_{1}(X) \rightarrow \pi_{1}(Y)$ is a monomorphism, then $\tilde{f}: \tilde{X} \rightarrow \tilde{Y}$ is an epimorphism of $\mathscr{H} \mathscr{C} \mathscr{W}^{*}$.

As a straightforward consequence the following results of Dyer-Roitberg (Topology Appl. (to appear)) is derived:

Theorem B. A map $f: X \rightarrow Y$ is an equivalence in $\mathscr{H} \mathscr{C} \mathscr{W}^{*}$, the homotopy category of pointed path-connected $C W$-spaces, iff it is both an epimorph.sm and a monomorphism in $\mathscr{H} \mathscr{C} \mathscr{W}^{*}$.

Recall that $f$ is a monomorphism (epimorphism) in $\mathscr{H} \mathscr{C} \mathscr{W}^{*}$ if given $\alpha, \beta: Z \rightarrow X(\alpha, \beta: Y \rightarrow Z), f \circ \alpha \approx f \circ \beta$ implies $\alpha \approx \beta \quad(\alpha \circ f \approx \beta \circ f$ implies $\alpha \approx \beta$ ).

Lemma 1. An inclusion $f: X \rightarrow Y$ is an epimorphism of $\mathscr{H} \mathscr{C} \mathscr{W}^{*}$ iff there is a map $H: Y \times[-1,1] \rightarrow(Y \times\{-1,1\} \cup X \times[-1,1])$ such that

$$
\begin{aligned}
H \mid Y & \times\{-1,1\} \cup\{*\} \times[-1,1]: \\
Y & \times\{-1,1\} \cup\{*\} \times[-1,1] \rightarrow Y \times\{-1,1\} \cup X \times[-1,1]
\end{aligned}
$$

is the inclusion.

Proof. Given two maps $g, h: Y \rightarrow Z$ such that $g \circ f \approx h \circ f$, there is $G: Y \times$ $\{-1,1\} \cup X \times[-1,1] \rightarrow Z$ such that $G|Y \times\{-1\}=g, G| Y \times\{1\}=h$, and $G \mid\{*\} \times I=$ const. Now, $G \circ H: Y \times[-1,1] \rightarrow Z$ is a homotopy joining $g$ and $h$, i.e., $f$ is an epimorphism of $\mathscr{H} \mathscr{C} \mathscr{W}^{*}$.

Suppose $f$ is an epimorphism of $\mathscr{H} \mathscr{C} \mathscr{W}^{*}$. Consider the two inclusion $j_{i}: Y \rightarrow(Y \times\{-1,1\} \cup X \times[-1,1]) /\{*\} \times[-1,1], j_{i}(y)=(y, i), i=-1,1$, and notice that $j_{-1} \circ f \approx j_{1} \circ f$. Hence, $j_{-1} \approx j_{1}$; i.e., there is $H^{\prime}: Y \times[-1,1] \rightarrow$ $(Y \times\{-1,1\} \cup X \times[-1,1]) /\{*\} \times[-1,1]$ such that $H^{\prime}(y, i)=(y, i)$ for $(y, i) \in Y \times\{-1,1\}$. Since the projection $Y \times\{-1,1\} \cup X \times[-1,1] \rightarrow$ $(Y \times\{-1,1\} \cup X \times[-1,1]) /\{*\} \times[-1,1]$ is a homotopy equivalence, Lemma 1

Received by the editors April 15, 1991.

1991 Mathematics Subject Classification. Primary 55N25, 55P10.

Key words and phrases. Homotopy-epimorphisms, homotopy-monomorphisms, homotopy equivalences.

Supported in part by an NSF grant. 
follows (the map $Y \times\{-1,1\} \cup\{*\} \times[-1,1] \rightarrow(Y \times\{-1,1\} \cup X \times[-1,1]) /\{*\} \times$ $[-1,1]$ extends over $Y \times[-1,1]$, so the inclusion $Y \times\{-1,1\} \cup\{*\} \times[-1,1] \rightarrow$ $Y \times\{1,1\} \cup X \times[-1,1]$ extends over $Y \times[-1,1])$.

Theorem A. If $f: X \rightarrow Y$ is an epimorphism of $\mathscr{H} \mathscr{C} \mathscr{W}^{*}$, the homotopy category of pointed path-connected $C W$-spaces and if $\pi_{1}(f): \pi_{1}(X) \rightarrow \pi_{1}(Y)$ is a monomorphism, then $\tilde{f}: \widetilde{X} \rightarrow \widetilde{Y}$ is an epimorphism of $\mathscr{H} \mathscr{C} \mathscr{W}^{*}$.

Proof. For simplicity, assume $X \subset Y$ and $f$ is the inclusion (replace $f$ by the inclusion $X \rightarrow M(f)$ from $X$ to the reduced mapping cylinder of $f$ ). Let $\pi: \widetilde{Y} \rightarrow Y$ be the projection from the universal cover of $Y$ to $Y$. Choose a base point $*$ in $\pi^{-1}(*)$. Notice that $p=\pi \times$ id: $\widetilde{Y} \times[-1,1] \rightarrow Y \times[-1,1]$ is the universal covering projection. If $\pi_{1}(f)$ is an isomorphism, then $\pi^{-1}(X)$ is the universal cover $\widetilde{X}$ of $X$ and $p^{-1}(Y \times\{-1,1\} \cup X \times[-1,1])=\widetilde{Y} \times$ $\{-1,1\} \cup \widetilde{X} \times[-1,1]$ is the universal cover of $Y \times\{-1,1\} \cup X \times[-1,1]$ as the inclusion $Y \times\{-1,1\} \cup X \times[-1,1] \rightarrow Y \times[-1,1]$ induces an isomorphism of fundamental groups. Choose the lift $\widetilde{H}: \widetilde{Y} \times[-1,1] \rightarrow \widetilde{Y} \times\{-1,1\} \cup \widetilde{X} \times[0,1]$ of $H$ with $\widetilde{H}(* \times\{-1\})=\{*\}$. Clearly, $\widetilde{H} \mid \widetilde{Y} \times\{-1,1\}=$ id, which proves that $\tilde{f}$ is an epimorphism of $\mathscr{H} \mathscr{C} \mathscr{W}^{*}$.

Lemma 2. If $f: X \rightarrow Y$ is an epimorphism of $\mathscr{H} \mathscr{C} \mathscr{W}^{*}$, then

(a) $\pi_{1}(f): \pi_{1}(X) \rightarrow \pi_{1}(Y)$ is an epimorphism,

(b) $H_{k}(f): H_{k}(X) \rightarrow H_{k}(Y)$ is an epimorphism for all $k \geq 1$,

Proof. For simplicity, assume $X \subset Y$ and $f$ is the inclusion (replace $f$ by the inclusion $X \rightarrow M(f)$ from $X$ to the reduced mapping cylinder of $f$ ). Notice that $\pi_{1}((Y \times\{-1,1\} \cup X \times[-1,1]) /\{*\} \times[-1,1])$ is the amalgamated product of two copies of $\pi_{1}(Y)$, with two copies of $\operatorname{im}\left(\pi_{1}(X) \rightarrow \pi_{1}(Y)\right)$ identified. The existence of $H: Y \times[-1,1] \rightarrow(Y \times\{-1,1\} \cup X \times[-1,1]) /\{*\} \times[-1,1]$ as in Lemma 1 means that

$$
\pi_{1}(Y \times\{-1\})=\pi_{1}(Y \times\{1\})
$$

in $\pi_{1}((Y \times\{-1,1\} \cup X \times[-1,1]) /\{*\} \times[-1,1])$, which is possible only if $\pi_{1}(f)$ is an epimorphism. This proves $(\mathbf{a})$.

Consider the projection $\pi: Y \rightarrow Y / X$ and the trivial map $c: Y \rightarrow Y / X$. Since $\pi \circ f=c \circ f$, it follows that $\pi \approx c$. From the homology sequence $H_{k}(X) \rightarrow H_{k}(Y) \rightarrow H_{k}(Y / X) \rightarrow \cdots$ we deduce (b).

Corollary. If $f: X \rightarrow Y$ is an epimorphism of $\mathscr{H} \mathscr{C} \mathscr{W}^{*}$ and $\pi_{k}(f)$ is a monomorphism for all $k \geq 1$, then $f$ is an equivalence of $\mathscr{H} \mathscr{C} \mathscr{W}^{*}$.

Proof. It suffices to show that $\pi_{k}(f)$ is an isomorphism for all $k \geq 1$. By the above result $\pi_{1}(f)$ is an isomorphism and we may assume (by switching to universal covers) that $X$ and $Y$ are simply connected. Suppose $\pi_{n}(Y, X)=0$ for all $n \leq m$. Then, $\pi_{m+1}(Y, X) \approx H_{m+1}(Y, X)$ and from the diagram

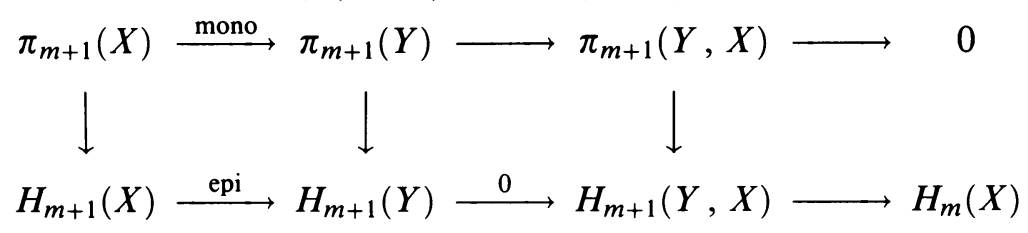

we conclude that $\pi_{m+1}(Y, X) \rightarrow H_{m+1}(Y, X)$ is trivial. Thus, $\pi_{n}(Y, X)=0$ for all $n \geq 1$ and $f$ is an equivalence. 
Now, the following result of Dyer and Roitberg [DR] (see [HR, $R_{1}, R_{2}$ ] for special versions of it) is a direct consequence of the Corollary:

Theorem B. A map $f: X \rightarrow Y$ is an equivalence in $\mathscr{H} \mathscr{C} \mathscr{W}^{*}$, the homotopy category of pointed path-connected $C W$-spaces, iff it is both an epimorphism and a monomorphism in $\mathscr{H} \mathscr{C} \mathscr{W}^{*}$.

\section{REFERENCES}

[DR] Eldon Dyer and Joseph Roitberg, Homotopy-epimorphisms, homotopy-monomorphisms and homotopy equivalences, Topology Appl. (to appear).

[HR] Peter Hilton and Joseph Roitberg, Relative epimorphisms and monomorphisms in homotopy theory, Compositio Math. 61 (1987), 353-367.

[R1] Joseph Roitberg, Monomorphisms and epimorphisms in homotopy theory, Israel J. Math. 46 (1983), 205-211.

[R2] - Residually finite, Hopfian and co-Hopfian spaces, Contemp. Math., vol. 37, Amer. Math. Soc., Providence, RI, 1985, pp. 131-144.

Department of Mathematics, University of Tennessee, Knoxville, Tennessee 37996 E-mail address: dydak@utkvx.bitnet 